\title{
BMJ Open What is a public health approach to substance use? Protocol for a qualitative systematic review
}

\author{
Jean-François Crépault (ib , ${ }^{1,2}$ Tara Marie Watson, ${ }^{1}$ Carol Strike, ${ }^{2}$ Sarah Bonato, ${ }^{1}$ \\ Jürgen Rehm ${ }^{1,2}$
}

\begin{abstract}
To cite: Crépault J-F, Watson TM, Strike C, et al. What is a public health approach to substance use? Protocol for a qualitative systematic review. BMJ Open 2021;11:e055991. doi:10.1136/ bmjopen-2021-055991
\end{abstract}

- Prepublication history and additional supplemental material for this paper are available online. To view these files, please visit the journal online (http://dx.doi.org/10.1136/ bmjopen-2021-055991).

Received 02 August 2021 Accepted 23 September 2021

Check for updates

(c) Author(s) (or their employer(s)) 2021. Re-use permitted under CC BY-NC. No commercial re-use. See rights and permissions. Published by BMJ.

${ }^{1}$ Centre for Addiction and Mental Health, Toronto, Ontario, Canada 2Dalla Lana School of Public Health, University of Toronto, Toronto, Ontario, Canada

Correspondence to Jean-François Crépault; jeanfrancois.crepault@camh.ca

\section{ABSTRACT}

Introduction The concept of a 'public health approach' to substance use is frequently but inconsistently invoked. This inconsistency is reflected in public policy, with governments using the term 'public health approach' in contradictory ways. This aim of this study is to clarify what is meant and understood when the term 'public health approach' is used in the context of substance use.

Methods and analysis We will conduct a systematic search of Medline, Embase, Scopus, CINAHL, PsycINFO, Sociological Abstracts and PAIS Index. Eligible articles will be from peer-reviewed journals, in English, with full text available. There will be no limits on year of publication. Substance use must be the primary topic of the article. Editorials, commentaries and letters to the editor will be included, but not commentaries on other articles, unless the definition of a public health approach is central to the commentary. Data selection and collection will be conducted independently by two researchers, with a third separately resolving any disagreement. To answer the research question, we will extract authors' definitions of a public health approach to substance use as well as any descriptions of the central principles, characteristics and components of such an approach. To synthesise the data, we will employ thematic synthesis. Coding will be conducted by one researcher and verified by a second; two researchers will then group the codes into themes using an inductive process. Finally, the full research team will develop a set of analytic themes, which will be presented as a narrative.

Ethics and dissemination Ethics approval is not needed since the research will only involve published work. Our findings will be disseminated in a peer-reviewed journal and, if possible, at conferences.

PROSPERO registration number CRD42021270632.

\section{INTRODUCTION}

The use of psychoactive substances, while common, comes with risks. It has been estimated that $5.5 \%$ of disability-adjusted lifeyears globally can be attributed to substance use. ${ }^{1}$ WHO reports that 3 million deaths per year can be attributed to alcohol and a further 500000 to illicit drugs. ${ }^{23}$ Drug policy can cause harm as well; in much of the world, the commercialisation of alcohol and the criminalisation of opioids have exacerbated

\section{Strengths and limitations of this study}

This review will allow the development of a practical definition of a public health approach to substance use that will be of value to policy-makers.

- We will employ thematic synthesis, which was specifically designed to rigorously and transparently synthesise research in public health/health promotion.

- Limiting to English-language literature means we will miss debate from much of the world.

- This review is unlikely to shed light on the impact or outcomes of measures associated with a public health approach to substance use.

the health risks of those substances and introduced further social harms. ${ }^{45}$

The concept of a 'public health approach' to substance use is frequently invoked in the English-language academic literature, but definitions are often vague. There are papers calling for such an approach that make no attempt to define it. ${ }^{67}$ Where definitions are offered, they vary greatly. For instance, some calls for a public health approach focus on substance use disorders as fully preventable brain disease that should be addressed as health rather than criminal issues, primarily through evidence-based prevention and treatment interventions. ${ }^{8}$ Others rest on the premise that some level of substance use in society is inevitable, and include the reduction of harm, support for human rights, and the facilitation of participatory democracy as fundamental principles. ${ }^{9}$

This lack of a consistent definition is reflected in public policy. The term 'public health approach' is widely used in public and policy-maker debates. For instance, Canada's federal government frequently states that it is committed to a public health approach to cannabis, opioids and substance use more broadly. ${ }^{10-12}$ Yet the government's cannabis and opioids policies differ markedly. It is 
difficult to discern many commonalities between Canada's approach to cannabis, legalised and regulated since 2018 , and its approach to opioids, in which criminal justice continues to play a major role. It has also been observed that globally, many countries are using the term 'public health approach' to describe their drug policy, using it as a 'smokescreen' to obscure policies that are coercive and may be contrary to 'good public health practice'. ${ }^{13}$

\section{OBJECTIVE}

This study will systematically review the English-language academic literature in order to understand what is meant and understood when the term 'public health approach' is used in the context of substance use. Using a modified form of the PICo tool for formulating qualitative systematic review questions (see online supplemental material, p. 1 , for a brief discussion), ${ }^{14}$ the problem is substance use/drug policy, the phenomenon of interest is definitions of a public health approach, and the context is the English-language academic literature. Thus, our research question is: How is a public health approach to substance use defined and described in the English-language academic literature?

\section{METHODS AND ANALYSIS \\ Eligibility criteria}

Eligible articles will be from peer-reviewed journals, in the English language, with full text available. Grey literature will be excluded from this review (see online supplemental material, p. 2, for rationale). There will be no limits on year of publication. Editorials, commentaries and letters to the editor will be included, but not responses to or commentaries on other articles, unless the definition of a public health approach is central to the response or commentary. Substance use must be the primary topic of the article-for example, an article about public health approaches to HIV that secondarily discusses intravenous drug use would be excluded. Similarly, an article proposing a public health approach to gambling that draws on the substance use literature would also be excluded.

\section{Information sources and search strategy}

We will use the following academic search engines: Medline, Embase, Scopus, CINAHL, PsycINFO, Sociological Abstracts and PAIS Index. We will use text word and subject heading terms, depending on the database, employing keywords for the phenomenon of interest-public health approaches to substance use. A search strategy has been created for Medline (see online supplemental material, p. 3); it will be adapted to the other six databases. We will also check the reference lists of included articles for potentially relevant sources that our search may have missed.

\section{Data management and selection process}

To facilitate the screening process we will use the Covidence systematic review software. ${ }^{15}$ We will record details of all searches. Results will be entered into Covidence and deduplicated. Two reviewers will independently review titles and abstracts against an eligibility checklist (for criteria, see above), with a third person separately resolving any disagreement. They will then review full-text articles for eligibility, with a third researcher again resolving differences, and eliminate any remaining duplicates. This process, like the rest of the review, will be reported using the most recent Preferred Reporting Items for Systematic Reviews and Meta-Analyses guidelines. ${ }^{16}$

\section{Data collection process, data items}

Two reviewers will independently extract data using an extraction form (see online supplemental material, p. 4, for a draft) and enter them into qualitative data analysis software NVivo ${ }^{17}$; any differences will be resolved by consensus or, if necessary, by a third team member. The following descriptive information about the included articles will be extracted for context: full reference, article type (commentary, review, etc), country of origin, substance(s) under discussion, and author funding (if applicable). To answer the research question, we will extract authors' definitions of a public health approach to substance use (or particular substances), as well as any descriptions of the central principles, characteristics and components of such an approach. Negative definitions (ie, what a public health approach is not) will also be extracted. These will need to be explicitly stated by the authors; if no definition or characteristics are offered, we will not attempt to infer them, but the study will be included and the lack of a definition recorded. We will also record any sources the authors may have cited in support of their definition of a public health approach to substance use. The data extraction table included in this article's online supplemental material gives an example of the data items collected for one article returned in our test search.

\section{Risk of bias in individual studies}

Assessing study quality and risk of bias is an important part of qualitative systematic reviews. ${ }^{18}{ }^{19}$ Generally, this is accomplished 'by focusing on assessment of methodological strengths and limitations as a marker of study rigour' ${ }^{19}$ However, this systematic review is different from most: its sources will generally not be research studies, and the data to be extracted-definitions of a public health approach-will be expert opinion rather than research findings. While we are aware of no guidelines for assessing risk of bias when the object of a systematic review is expert opinion, we will track author funding in order to explore the possibility of bias. Study quality will not be assessed. 


\section{Synthesis}

Given the subject matter, synthesis will be entirely qualitative. This review will employ thematic synthesis, designed by Thomas and Harden as a way to rigorously and transparently synthesise research in the area of health promotion. ${ }^{19-21}$ Thematic synthesis has three stages of analysis: (1) coding text, (2) grouping codes into descriptive themes and (3) interpreting those to generate analytic themes. ${ }^{21}$

Coding will be conducted by one researcher using NVivo. A second researcher will verify the coding; disagreements will be resolved by consensus or, failing that, by a third team member. Two researchers will then group the codes into themes, using the inductive process outlined above, and prepare a draft for the other team members outlining these descriptive themes and offering an initial interpretation.

The final stage of thematic synthesis is the generation of analytic themes. The objective here is to 'go beyond' the primary studies and generate new interpretive constructs, explanations or hypotheses." ${ }^{21}$ In this stage, researchers generally work independently at first, then as a group, repeating as many times as necessary to reach consensus on a set of analytic themes. ${ }^{21-23}$ The full team will participate in this process. In the interest of transparency, a detailed account will be recorded and reported.

Overall results will be summarised in tables. Analytical themes will be presented as a narrative, with the support of additional tables and infographics as needed. The full team will participate in this process as well.

\section{Meta-Bias}

In most systematic reviews, it is necessary to assess possible meta-bias, including selective reporting and publication bias. ${ }^{18}$ In this case, we are intentionally excluding grey literature for the reasons outlined in online supplemental material (p. 2), and since we are examining only the English-language literature, we are also aware that the review will fail to capture drug policy debate from much of the world. However, we believe this will not constitute metabias in the context of our research question (How is a public health approach to substance use defined and described in the English-language academic literature?).

\section{Confidence in cumulative evidence}

To assess confidence in our review findings, the research team will use the Grades of Recommendations, Assessment, Development and EvaluationConfidence in the Evidence from Reviews of Qualitative research tool, designed to assess 'the extent to which a review finding is a reasonable representation of the phenomenon of interest. ${ }^{24}$ It does so through an assessment of methodological limitations, coherence, adequacy of data and relevance for each individual review finding.

\section{Patient and public involvement}

There is no planned patient or public involvement in the conduct of this systematic review.

\section{ETHICS AND DISSEMINATION}

Ethics approval is not needed since the research will only involve published work. Our findings will be disseminated in a peer-reviewed journal and, if possible, at conferences.

Contributors JFC conceptualised the project and drafted the protocol. TMW, CS, $\mathrm{SB}$ and JR critically reviewed it and provided feedback. JFC is the guarantor. All approved the final manuscript.

Funding The authors have not declared a specific grant for this research from any funding agency in the public, commercial or not-for-profit sectors.

Competing interests None declared.

Patient consent for publication Not applicable.

Provenance and peer review Not commissioned; externally peer reviewed.

Supplemental material This content has been supplied by the author(s). It has not been vetted by BMJ Publishing Group Limited (BMJ) and may not have been peer-reviewed. Any opinions or recommendations discussed are solely those of the author(s) and are not endorsed by BMJ. BMJ disclaims all liability and responsibility arising from any reliance placed on the content. Where the content includes any translated material, BMJ does not warrant the accuracy and reliability of the translations (including but not limited to local regulations, clinical guidelines, terminology, drug names and drug dosages), and is not responsible for any error and/or omissions arising from translation and adaptation or otherwise.

Open access This is an open access article distributed in accordance with the Creative Commons Attribution Non Commercial (CC BY-NC 4.0) license, which permits others to distribute, remix, adapt, build upon this work non-commercially, and license their derivative works on different terms, provided the original work is properly cited, appropriate credit is given, any changes made indicated, and the use is non-commercial. See: http://creativecommons.org/licenses/by-nc/4.0/.

ORCID iD

Jean-François Crépault http://orcid.org/0000-0003-0448-560X

\section{REFERENCES}

1 GBD 2016 Alcohol and Drug Use Collaborators. The global burden of disease attributable to alcohol and drug use in 195 countries and territories, 1990-2016: a systematic analysis for the global burden of disease study 2016. Lancet Psychiatry 2018;5:987-1012.

2 World Health Organization. Opioid overdose, 2020. Available: https:// www.who.int/news-room/fact-sheets/detail/opioid-overdose

3 World Health Organization. Alcohol, 2021. Available: https://www. who.int/health-topics/alcohol\#tab=tab_1

4 Anderson P, Braddick F, Conrod PJ. The new governance of addictive substances and behaviours. Oxford, UK: Oxford University Press, 2017.

5 Babor TF, Caulkins J, Fischer B. Drug policy and the public good. $2^{\text {nd }}$ ed. Oxford, UK: Oxford University Press, 2019.

6 Lancet Public Health. A public health approach to Canada's opioid crisis. Lancet Public Health 2018;3:e204.

7 Shokoohi M, Rahimi-Movaghar A, Noroozi A, et al. A public health approach to alcohol use and its related harms in Iran. Lancet Public Health 2019;4:e175-6.

8 Volkow ND, Poznyak V, Saxena S, et al. Drug use disorders: impact of a public health rather than a criminal justice approach. World Psychiatry 2017;16:213-4.

9 Emerson B, Haden M. A public health based vision for the management and regulation of opioids. Int $J$ Drug Policy 2021;91:103201.

10 Health Canada. Health Canada releases summary of comments from cannabis regulatory consultations, 2018. Available: https://www. canada.ca/en/health-canada/news/2018/03/health-canada-releasessummary-of-comments-from-cannabis-regulatory-consultations.html

11 Health Canada. Government of Canada begins consultation to better ensure the continuity of supervised consumption sites and services in Canada, 2020. Available: https://www.canada.ca/en/health- 
canada/news/2020/08/government-of-canada-begins-consultationto-better-ensure-the-continuity-of-supervised-consumption-sitesand-services-in-canada.html

12 Health Canada. 2020-21 departmental plan: Health Canada, 2021. Available: https://www.canada.ca/en/health-canada/corporate/ transparency/corporate-management-reporting/report-planspriorities/2020-2021-report-plans-priorities.html

13 Csete J, Wolfe D. Seeing through the public health smoke-screen in drug policy. Int J Drug Policy 2017;43:91-5.

14 Munn Z, Stern C, Aromataris E, et al. What kind of systematic review should I conduct? A proposed typology and guidance for systematic reviewers in the medical and health sciences. BMC Med Res Methodol 2018;18:5.

15 Veritas Health Innovation.. Covidence systematic review software, 2021. Available: https://www.covidence.org

16 Page MJ, McKenzie JE, Bossuyt PM, et al. The PRISMA 2020 statement: an updated guideline for reporting systematic reviews. BMJ 2021;372:n71.

17 QSR International. NVivo qualitative data analysis software, 2020. Available: https://www.qsrinternational.com/nvivo-qualitative-dataanalysis-software/home
18 Shamseer L, Moher D, Clarke M, et al. Preferred reporting items for systematic review and meta-analysis protocols (PRISMA-P) 2015: elaboration and explanation. BMJ 2015;349:97647.

19 Noyes J, Booth A, Cargo M. Chapter 21: Qualitative evidence. In: Cochrane Handbook for systematic reviews of interventions version 6.1 (updated September 2020. Higgins ed, 2020. https://training. cochrane.org/cochrane-handbook-systematic-reviews-interventions

20 Barnett-Page E, Thomas J. Methods for the synthesis of qualitative research: a critical review. BMC Med Res Methodol 2009;9:59.

21 Thomas J, Harden A. Methods for the thematic synthesis of qualitative research in systematic reviews. BMC Med Res Methodol 2008;8:45.

22 Houghton C, Dowling M, Meskell P, et al. Factors that impact on recruitment to randomised trials in health care: a qualitative evidence synthesis. Cochrane Database Syst Rev 2020;10:MR000045.

23 Passey ME, Longman JM, Robinson J, et al. Smoke-Free homes: what are the barriers, motivators and enablers? A qualitative systematic review and thematic synthesis. BMJ Open 2016;6:e010260.

24 Lewin S, Bohren M, Rashidian A, et al. Applying GRADE-CERQual to qualitative evidence synthesis findings-paper 2: how to make an overall CERQual assessment of confidence and create a summary of qualitative findings table. Implement Sci 2018;13:10. 